\title{
Criterion validity of The International Physical Activity Questionnaire-Short Form (IPAQ-SF) for use in clinical practice in patients with osteoarthritis
}

Kenth Louis Joseph ${ }^{1,2^{*}}$ D, Hanne Dagfinrud ${ }^{1}$, Anne Christie ${ }^{1}$, Kåre Birger Hagen ${ }^{1,3}$ and Anne Therese Tveter ${ }^{1}$

\begin{abstract}
Background: To tailor physical activity treatment programs for patients with osteoarthritis, clinicians need valid and feasible measurement tools to evaluate habitual physical activity. The widely used International Physical Activity Questionnaire-Short Form (IPAQ-SF) is not previously validated in patients with osteoarthritis.

Purpose: To assess the concurrent criterion validity of the IPAQ-SF in patients with osteoarthritis, using an accelerometer as a criterion-method.

Method: Patients with osteoarthritis $(n=115)$ were recruited at The Division of Rheumatology and Research at Diakonhjemmet Hospital (Oslo, Norway). Physical activity was measured by patients wearing an accelerometer (ActiGraph wGT3X-BT) for seven consecutive days, followed by reporting their physical activity for the past 7 days using the IPAQ-SF. Comparison of proportions that fulfilled physical activity recommendations as measured by the two methods were tested by Pearson Chi-Square analysis. Differences in physical activity levels between the IPAQSF and the accelerometer were analyzed with Wilcoxon Signed-Rank Test and Spearman rank correlation test. Bland-Altman plots were used to visualize the concurrent criterion validity for total- and intensity-specific physical activity levels.

Results: In total, 93 patients provided complete physical activity data, mean (SD) age was 65 (8.7) years, 87\% were women. According to the IPAQ-SF, 57\% of the patients fulfilled the minimum physical activity recommendations compared to $31 \%$ according to the accelerometer $(p=0.043)$. When comparing the IPAQ-SF to the accelerometer we found significant under-reporting of total physical activity MET-minutes $(p=<0.001)$, sitting $(p=<0.001)$ and walking $(p<0.001)$, and significant over-reporting of moderate-to-vigorous physical activity $(p<0.001)$. For the different physical activity levels, correlations between the IPAQ-SF and the accelerometer ranged from rho 0.106 to 0.462. The Bland-Altman plots indicated an increased divergence between the two methods with increasing time spent on moderate-to-vigorous intensity physical activity.

\footnotetext{
* Correspondence: Kenth-Louis.Joseph@diakonsyk.no

'National Advisory Unit on Rehabilitation in Rheumatology, The Division of Rheumatology and Research, Diakonhjemmet Hospital, Oslo, Norway

${ }^{2}$ Faculty of Medicine, Institute of Health and Society, University of Oslo, Oslo, Norway

Full list of author information is available at the end of the article
}

C C The Author(s). 2021 Open Access This article is licensed under a Creative Commons Attribution 4.0 International License, which permits use, sharing, adaptation, distribution and reproduction in any medium or format, as long as you give appropriate credit to the original author(s) and the source, provide a link to the Creative Commons licence, and indicate if changes were made. The images or other third party material in this article are included in the article's Creative Commons licence, unless indicated otherwise in a credit line to the material. If material is not included in the article's Creative Commons licence and your intended use is not permitted by statutory regulation or exceeds the permitted use, you will need to obtain permission directly from the copyright holder. To view a copy of this licence, visit http://creativecommons.org/licenses/by/4.0/. The Creative Commons Public Domain Dedication waiver (http://creativecommons.org/publicdomain/zero/1.0/) applies to the data made available in this article, unless otherwise stated in a credit line to the data. 
(Continued from previous page)

Conclusion: Physical activity is a core treatment of osteoarthritis. Our finding that patients tend to over-report activity of higher intensity and under-report low-intensity activity and sitting-time is of clinical importance. We conclude that the concurrent criterion validity of the IPAQ-SF was weak in patients with osteoarthritis.

Keywords: Physical activity assessment, Criterion validity, IPAQ-SF, Accelerometer, Osteoarthritis, Clinical practice

\section{Background}

Tailored physical activity (PA) is the cornerstone in treatment of patients with osteoarthritis (OA) [1]. To curb disease specific symptoms like pain and joint stiffness as well as reduce the risk of cardiovascular comorbidities [1-5], PA of moderate-to-vigorous intensity is found effective and is recommended throughout the course of the disease [1, 6-8]. To provide individually tailored PA treatment programs with optimal exercise dosage, clinicians need valid and feasible measures to evaluate their patients' habitual PA level [9]. In clinical practice, the measurement tools must be easy to administer at a low cost and pose minimal patient burden, but still provide valid information.

PA is defined by requirement of increased energy expenditure [10]. PA energy expenditure in free-living environments can be assessed by the gold standard doubly labelled water method, by activity monitors, heart rate monitors, pedometers, diaries or by questionnaires [11]. The doubly labelled water method provides information solely on individuals' energy expenditure $[11,12]$. This method does not give information on habitual PA profile in terms of intensity, frequency or duration of the activity, which is important when evaluating patients' PA levels [1]. However, activity monitors such as accelerometers, provide daily profiles on habitual PA [11, 13]. Accelerometers also allow for calculation of PA energy expenditure and weekly time in intensity specific PA (e.g. according to PA recommendations). Accelerometers are shown to agree well with the doubly labelled water method and may therefore serve as a criterion-method for measuring habitual PA $[12,14,15]$.

Due to the lack of immediate information on PA from accelerometers, self-report questionnaires are often used in clinical practice. Questionnaires are easy to administer, have a low cost, and pose minimal burden on patients and clinicians, but a challenge is that they are prone to recall- and reporting bias $[16,17]$. A questionnaire that is frequently used in research and clinical practice is The International Physical Activity Questionnaire-Short Form (IPAQ-SF) [18-21]. The IPAQ-SF was originally developed and validated for population surveys [22]. The validity of the questionnaire has been assessed in some disease-groups [19], but evidence on criterion validity in patients with $\mathrm{OA}$ is lacking [18, 20, 21]. Knowledge about criterion validity is important when assessing PA [20, 21], and in the recommendations for PA in people with OA and inflammatory arthritis the need for valid and feasible methods for assessing PA in clinical practice is underlined [1]. Thus, the purpose of the present study was to assess the concurrent criterion validity of the IPAQ-SF in patients with OA using an accelerometer as a criterion-method.

\section{Methods \\ Subjects and design}

Patients who attended a one-day OA patient education program at The Division of Rheumatology and Research at Diakonhjemmet Hospital (Oslo, Norway) were consecutively recruited from November 2017 to June 2018. Attendance at the program required a referral confirming an OA diagnosis from a doctor at the hospital a or by their general practitioner. Individuals $\geq 18$ years of age, independent of walking aids and competent in verbal- and written Norwegian were eligible for inclusion. Exclusion criteria were patient's incapable of ambulatory movement and/or the inability to understand verbaland written Norwegian. Eligible patients were given verbal- and written information about the study, and those who provided written, informed consent were included. For excellent methodological quality, a minimum of 100 patients are recommended [23, 24]. To account for possible lack of compliance with using the accelerometer, a total of 115 patients were recruited.

Patients who consented to participate received a study pack including: the IPAQ-SF, an accelerometer, a diary on accelerometer wear-time, an instruction sheet on how to wear the accelerometer and a questionnaire on demographics.

Patients were instructed to wear the accelerometer mounted on the right hip (by an adjustable elastic belt) for all time awake except during water-based activities for seven consecutive days. On day six, a text message was sent to remind the patients to answer the questionnaires either on the seventh day before going to bed or in the morning on day eight, and to return the IPAQ-SF, the accelerometer, the wear-time diary, and the demographic questionnaire. in a pre-paid envelope. 


\section{The IPAQ-SF (the international physical activity} questionnaire - short form)

The previously translated Norwegian version of The IPAQ-SF (available at www.ipaq.ki.se) was used to assess self-reported PA [22]. The IPAQ-SF addresses the number of days and time spent on PA in moderate intensity, vigorous intensity and walking of at least 10-min duration the last 7 days, and also includes time spent sitting on weekdays the last 7 days [22]. The IPAQ-SF sum score is expressed in PA Metabolic Equivalent of Task (MET)-minutes per day or week. In the present study, data processing and analysis were calculated according to the official IPAQ scoring protocol (www.ipaq.ki.se). Total weekly PA MET-minutes were estimated by adding up the calculated MET-minutes within each PA intensity level (moderate intensity $=4.0 \mathrm{MET}$, vigorous intensity = 8.0 MET and walking $=3.3 \mathrm{MET}$ ). The reported time spent on sitting was calculated as time per weekday. In addition, reported time on moderate and vigorous physical activity (MVPA) were summed and expressed as MVPA-minutes per week and as MVPA MET-minutes per week. Finally, to categorize patients fulfilling or not fulfilling the PA recommendations, MVPA-minutes $\quad(\geq 150$ or $<150 \quad$ MVPA-minutes per week) and vigorous intensity PA ( $\geq 75$ or $<75$ min per week) were dichotomized [1].

\section{The accelerometer}

The ActiGraph wGT3X-BT accelerometer (ActiGraph, LLC, Pensacola, FL) was used as a criterion measure of habitual PA. The accelerometer provides data on daily structure of habitual PA over 1-4 weeks, and is a frequently used and validated method [11, 12, 14, 15]. The tri-axial accelerometer continuously records ambulatory movement as counts per minute (CPM) were countthresholds correspond to different PA intensity levels $[13,25]$. Data were downloaded in 1-min time intervals obtained from the vertical axis using the associated licensed ActiLife software (version 6.13.3, ActiGraph, LLC). Valid PA registration was defined as minimum 4 days of at least $10 \mathrm{~h}$ of recording per day [25].

Total registration time was defined as $18 \mathrm{~h}$ of recording (from 6:00 a.m. to 00:00 a.m.) [26]. Non wear-time was defined as at least 60 consecutive minutes of zero counts (with allowance for $\leq 2 \mathrm{~min}$ with counts below $100)$, and wear-time was determined by subtracting non wear-time from $18 \mathrm{~h}$ [25]. Thresholds for different PA intensities were set to: $\geq 5999$ CPM (vigorous); $\geq 2020$ 5998 CPM (moderate); 100-2019 CPM (light) and $<100$ CPM (sedentary) [25].

The following calculations were done to make the accelerometer data comparable to the IPAQ-SF outcomes:
1) Total weekly time in different PA intensities was calculated by summing the recorded time within each threshold on valid days (in at least 10-min bouts, allowing for $\leq 2 \mathrm{~min}$ below the respective thresholds), then averaged over number of valid days, and finally multiplied by seven (1 week).

2) Total weekly PA MET-minutes were estimated by applying MET values congruent to the IPAQ-SF MET values, were the corresponding value for moderate intensity was 4.0 MET, vigorous intensity 8.0 MET and for light intensity 3.3 MET. Light activity (100-2019 CPM) was compared to IPAQSF walking. Sedentary time on valid weekdays was averaged over valid days and multiplied by 5 (weekdays).

3) Based on these variables, time on moderate and vigorous physical activity (MVPA) were summed and expressed as MVPA-minutes per week and MVPA MET-minutes per week.

4) Finally, to categorize patients fulfilling or not fulfilling the PA recommendations, MVPA-minutes $(\geq 150$ or $<150$ MVPA-minutes per week) and vigorous intensity PA ( $\geq 75$ or $<75$ min per week) were dichotomized [1].

The diary on accelerometer wear-time (hours on and off each day), included questions concerning activities such as swimming, bicycling/ergometer-cycling, resistance exercise (using weights/apparatus) and crossskiing/roller-skiing during the measurement week. The answer options on each activity were yes/no/don't know/don't remember (if yes; how many days and average time per day).

\section{Patients demographics}

Demographic characteristics were collected by selfreport, and comprised age (years), gender, height $(\mathrm{cm})$, weight (kg), smoking habits (current, previous, never), educational level (primary school, upper secondary school, $<4$ years college/university, $\geq 4$ years college/university), currently working (yes/no, if no; student, retired, well-fare, sick leave, else), civil status (living alone, living with someone), pain during the last week $(\mathrm{Nu}$ meric Rating Scale (NRS) ranging from ' 0 ' no pain to ' 10 ' worst imaginable pain) and general health (EQ-5D visual analog scale ranging from ' $O$ ' worst imaginable health to ' 100 ' best imaginable health). Specific OA joint was not recorded.

\section{Statistics}

Data are presented as frequency (n) and proportion (\%), mean and standard deviation (SD) or median and interquartile range (IQR, 25th and 75th percentile). Body Mass Index (BMI) was calculated by weight and height 
$\left(\mathrm{kg} / \mathrm{m}^{2}\right)$ and was categorized into normal weight $(\mathrm{BMI}<$ 25 ), overweight (BMI $\geq 25$ to $<30$ ) and obese (BMI $\geq 30$ ). Data on education level were dichotomized into $\geq 1$ year or $<1$ year of college/university. Those reporting 'yes' or 'no' on currently working were categorized as 'employed' and 'not employed', respectively (reporting 'yes' on currently working in combination with 'retired', 'sick leave' or 'else' was categorized as 'employed'). Pain was defined as none to mild pain (NRS $\leq 5)$ and moderate to severe pain (NRS $\geq 6$ ).

In our analyses, we included those with valid accelerometer data and complete data on moderate and vigorous PA by the IPAQ-SF. The difference between the corresponding IPAQ-SF and accelerometer measured PA levels were tested with Wilcoxon Signed-Rank Test. The mean differences and 95\% confidence interval (95\% $\mathrm{CI}$ that were calculated by independent t-test are shown. Proportions fulfilling the PA recommendations according to the two methods were compared by Pearson Chi-Square analysis.

Concurrent criterion validity of the IPAQ-SF against the corresponding accelerometer measured PA levels was analyzed by Spearman rank test. Correlation coefficients (rho) of $\leq 0.10$ were defined as negligible, $0.10-0.39$ as weak, 0.40 to 0.69 as moderate and $\geq 0.70$ as strong/very strong [27]. Based on previous studies, we hypothesized weak to moderate correlations between the different PA levels measured by IPAQ-SF and the accelerometer $[28,29]$. The mean difference in measured PA levels between the two methods was also visualized by Bland-Altman plots with 95\% limits of agreement [30].

Due to the accelerometers' limitations on recording activities such as bicycling, resistance exercises, skiing and swimming (the accelerometer is taken off during water-based activities), we examined if the discrepancy between the two methods in weekly MVPA-minutes were different between patients reporting and not reporting such activities using Mann-Whitney $U$ test. Significance levels were set to $P<0.05$ in all analyses. Statistical analyses were calculated using IBM SPSS Statistics Version 21.

\section{Results}

In total, 105 patients returned valid accelerometer data (Fig. 1), and $96 \%$ had five or more valid days with $\geq 10 \mathrm{~h}$ of recording per day. Mean (SD) wear-

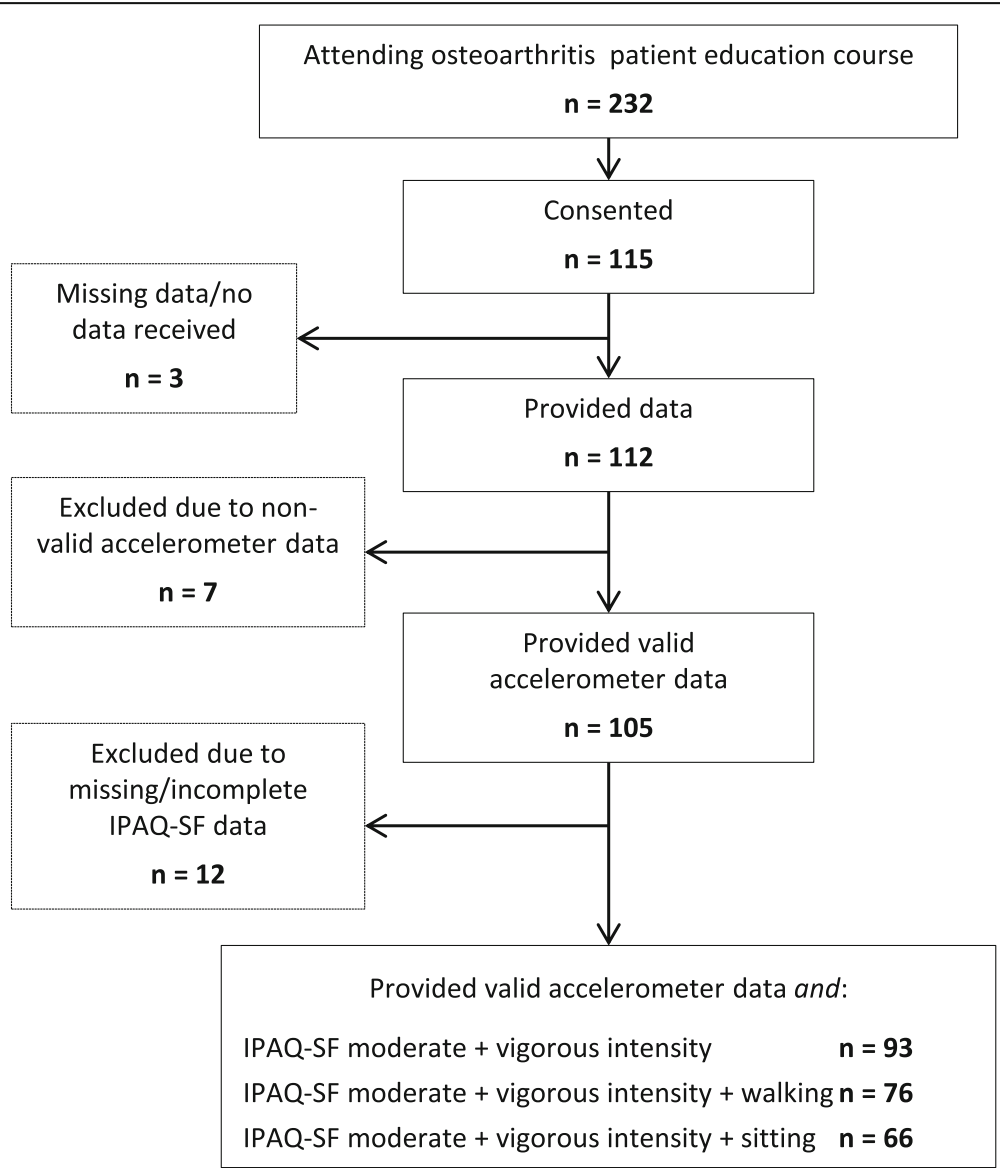

Fig. 1 Flowchart of patients that provided data and were included in the analyses 
time was 13.9 (1.2) hours per day. Among patients with valid accelerometer data, 93 (89\%) provided IPAQ-SF-data on both moderate and vigorous PA level (Fig. 1). The reasons for missing/incomplete PA data were mostly missing response and/or a 'don't know' response on moderate and/or vigorous intensity in the IPAQ-SF. No statistical differences were found in demographic characteristics between the 93 patients that were included for analyses and the 19 patients that had missing/incomplete PA data (data not shown).

Demographic characteristics are shown in Table 1. Half of the patients (49\%) were categorized as overweight/obese (BMI $\geq 25)$. Almost one in three $(28 \%)$ scored moderate to severe pain (NRS $\geq 6$ ).

More than half of the patients (57.0\%) fulfilled the PA recommendations (of minimum $150 \mathrm{~min}$ of at least moderate PA per week) according to IPAQ-data, while one in three (31.2\%) fulfilled the recommendations according to accelerometer data (Table 2).

When comparing PA levels measured with the two methods, significant differences were found for total PA MET-minutes and all intensity specific PA levels $(p<$ $0.001)$, except moderate intensity $(p=0.272)$. The patients reported lower total PA MET-minutes, less time sitting and walking, and more time in moderate and

Table 1 Demographic characteristics of patients with osteoarthritis, $n=93$

\begin{tabular}{|c|c|}
\hline Age (years), mean (SD) & $64.8(8.7)$ \\
\hline Women, n (\%) & $81(87.1)$ \\
\hline BMI $(\mathrm{kg} / \mathrm{m} 2)$, mean $(\mathrm{SD})^{\mathrm{a}}$ & $25.5(3.9)$ \\
\hline Normal weight (BMI < 25), n (\%) & $46(51.1)$ \\
\hline Overweight (BMI $\geq 25$ to <30), n (\%) & $32(35.6)$ \\
\hline Obese (BMI $\geq 30)$, n (\%) & $12(13.3)$ \\
\hline \multicolumn{2}{|l|}{ Smoking, n (\%) } \\
\hline Current & $8(8.6)$ \\
\hline Previous & $49(52.7)$ \\
\hline Never & $36(38.7)$ \\
\hline Education level $\geq 1$ year college/university, n (\%) & $63(67.7)$ \\
\hline Employed, $\mathrm{n}(\%)^{\mathrm{b}}$ & $42(46.2)$ \\
\hline \multicolumn{2}{|l|}{ Not employed status, n (\%) } \\
\hline Retired & $37(40.6)$ \\
\hline Well-fare/sick leave/else & $12(13.2)$ \\
\hline Civil status, living alone & $36(38.7)$ \\
\hline Pain (NRS, 0-10), mean (SD) ${ }^{c}$ & $4.3(2.1)$ \\
\hline General health (EQ-5D-VAS, 0-100), mean (SD) & $64.3(18.8)$ \\
\hline
\end{tabular}

vigorous PA compared to what was measured by the accelerometer (Table 2). The correlation between PA levels from the IPAQ-SF and the corresponding PA levels from the accelerometer ranged from rho 0.106 to 0.462 , were sitting time correlated moderately, and total PA METminutes and all intensity specific PA levels correlated weakly (Table 3). The Bland-Altman plots visualized that the difference between the methods increased with increasing minutes of PA for moderate intensity, MVPAminutes and MVPA MET-minutes (Fig. 2, b, c, e).

Finally, 59 patients reported to spend a median (IQR) of $120(60,222)$ minutes on one or more activities inadequately captured by the accelerometer (cycling, resistance exercises, skiing or swimming). However, for the difference between IPAQ-SF and accelerometer measured time spent on MVPA, no statistical betweengroup difference was found $(p=0.560)$.

${ }^{\mathrm{a}}, n=76$ due to incomplete response on IPAQ-SF.

b, $n=66$ due to in incomplete response on IPAQ-SF.

c, $p$-value for difference calculated by Pearson ChiSquare analysis.

${ }^{\mathrm{d}}$, p-value for difference calculated by two-tailed paired Wilcoxon Signed Ranks Test.

e, p-value by Wilcoxon analysis not included because calculations resulted in 44 ties with zero vigorous minutes between the IPAQ-SF and the accelerometer.

${ }^{\mathrm{a}}, n=76$ due to incomplete response on IPAQ-SF.

b, $n=66$ due to incomplete response on IPAQ-SF.

\section{Discussion}

In the present study, we compared self-reported physical activity (IPAQ-SF) with concurrent, objectively measured activity (accelerometer) in patients with OA. The main findings were that the patients overestimated self-reported moderate and vigorous activity and underestimated light activity, sitting time and total PA MET-minutes compared to data obtained with the accelerometer.

Based on self-reported PA, we found that more than half of the patients fulfilled the PA recommendations, but only one third of the patients were sufficiently active according to data from the accelerometer. In clinical practice it is important to identify patients that do not fulfill the PA recommendations. However, as our results indicate, these patients may not necessarily be identified by self-reporting their physical activity, which may result in sub-optimal disease management and increased risk of comorbidity.

In our study, we found weak correlations between selfreported and objectively measured total PA MET minutes and the different PA levels. This is in line with results from studies in individuals with self-reported OA [29], hip OA [31], hip- or knee arthroplasty [28] and in the general population [26], showing similar weak to moderate correlations between various self-reported and 
Table 2 Physical activity values assessed by IPAQ-SF and accelerometer, including $p$-values for differences $(n=93)$

\begin{tabular}{|c|c|c|c|c|}
\hline & IPAQ-SF & Accelerometer & Mean difference $(95 \% \mathrm{Cl})$ & $\begin{array}{l}\text { Difference } \\
p \text {-value }\end{array}$ \\
\hline \multicolumn{5}{|l|}{ PA guidelines*, $n$ (\%) } \\
\hline Proportion fulfilling PA guidelines & $53(57.0)$ & $29(31.2)$ & & $0.043^{c}$ \\
\hline \multicolumn{5}{|l|}{ PA levels, median (IQR) } \\
\hline Total PA MET-minutes, per week & $1985(898,4217)^{a}$ & $4059(2712,5467)$ & $-1616(-2096,-1137)$ & $<0.001^{d}$ \\
\hline MVPA MET-minutes, per week & $780(120,1680)$ & $238(45,648)$ & $775(445,1104)$ & $<0.001^{d}$ \\
\hline MVPA-minutes, per week & $180(30,300)$ & $60(11,162)$ & $118(53,183)$ & $<0.001^{d}$ \\
\hline Vigorous PA, minutes per week & $15(0,120)$ & $0(0,0)$ & $70(45,96)$ & - e \\
\hline Moderate PA, minutes per week & $90(0,210)$ & $60(11,162)$ & $53(-1,107)$ & $0.272^{d}$ \\
\hline Walking/light PA, minutes per week & $245(105,630)^{a}$ & $1101(711,1475)$ & $-729(-865,-593)$ & $<0.001^{d}$ \\
\hline Sitting/sedentary time, hours per weekday & $6.0(4.0,10.0)^{b}$ & $8.9(7.8,10.0)$ & $-1.8(-2.5,-1.1)$ & $<0.001^{d}$ \\
\hline
\end{tabular}

95\% Cl, 95\% confidence interval; IPAQ-SF The International Physical Activity Questionnaire-Short Form; MVPA moderate to vigorous intensity physical activity; MET Metabolic Equivalent of Task.

Data given in frequency (percent) for 'Proportion fulfilling PA guidelines' and median (interquartile range, IQR) for 'PA levels'.

*Fulfilling PA guidelines, $\geq 150$ min of MVPA or $\geq 75$ min of vigorous PA per week

objectively measured PA levels. Our results underline that PA measures from the two methods cannot be used interchangeably. Important findings of our study were also that participants' under-reported total PA METminutes and time spent on light activities, while they reported three times more MVPA-minutes than recorded by the accelerometer. Further, this discrepancy increased with more self-reported time in MVPA. Divergence between the methods can be explained by recall- and reporting bias related to use of questionnaires [16]. For example, walking is an everyday, "unconscious" activity that can be difficult to report in detail, whereas the accelerometer records walking with high accuracy [32]. The underreporting of time spent on walking may indicate that people pay less attention to light, every-day activity than to more intensive activity. Another possible explanation may be that walking is experienced as strenuous in patients with pain and stiffness due to hipand knee $\mathrm{OA}$, and therefore reported as more vigorous than shown by accelerometer. Since walking is underreported and MVPA over-reported, the results in our

Table 3 Spearman correlations (rho) with $p$-values between IPAQ-SF and accelerometer measured PA levels, $n=93$

\begin{tabular}{|c|c|c|}
\hline PA levels & Correlation & $p$-value \\
\hline Total PA MET-minutes, per week ${ }^{a}$ & 0.373 & 0.001 \\
\hline MVPA MET-minutes, per week & 0.315 & 0.002 \\
\hline MVPA-minutes, per week & 0.329 & 0.001 \\
\hline Vigorous PA, minutes per week & 0.106 & 0.311 \\
\hline Moderate PA, minutes per week & 0.275 & 0.008 \\
\hline Walking/light PA, minutes per week ${ }^{a}$ & 0.145 & 0.210 \\
\hline Sitting/sedentary time, hours per weekday ${ }^{b}$ & 0.462 & $<0.001$ \\
\hline
\end{tabular}

study underline that recording solely total PA minutes is not sufficient when evaluating patients' habitual PA.

Pain is a predominant symptom in OA [2], and patients may therefore prefer to engage in moderate to vigorous activities that cause less joint-related pain, i.e. resistance exercises, bicycling or swimming. However, such activities are inadequately recorded by a hip-worn accelerometer. We hypothesized that the type of activity could explain some of the discrepancy between the two methods in our study, but statistical analyses rejected this hypothesis, as no difference in over-reporting of MVPA-minutes was found between those engaging and not engaging in such activities.

In the present study, we found that $57 \%$ of the patients fulfilled the PA recommendations based on data from the IPAQ-SF, whereas only $31 \%$ was sufficiently active based on data from the accelerometer. Similar results are shown in a population-based OA study, in which half of the patients reported PA levels meeting the recommendations compared to only $<15 \%$ according to data from accelerometers [29]. Clinicians must be aware that even if patients report adequate PA, they may not really meet the PA recommendations. Sufficiently dosed PA is shown to be an effective treatment alternative for patients with musculoskeletal diseases, leading to less pain, improved physical function [6-8] and improved cardiorespiratory fitness which in turn is associated with reduced risk of cardiovascular disease [1, 33]. Thus, identifying patients not fulfilling the PA recommendations is important to provide optimal disease management for this large group of patients.

Our study has some limitations. A potential selection bias may be present as patients attending an educational course probably have a more positive attitude towards and are more likely to adhere to treatment 


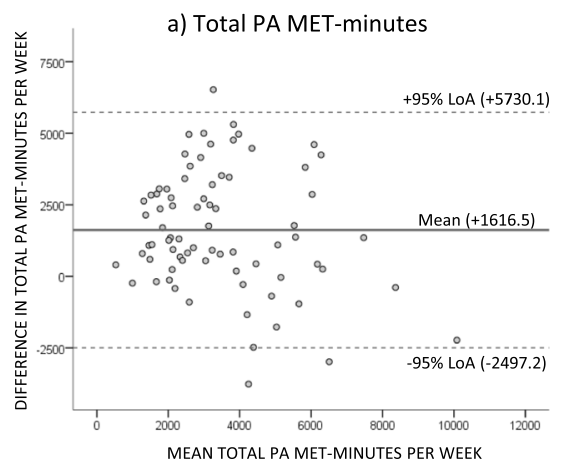

b) MVPA MET-minutes
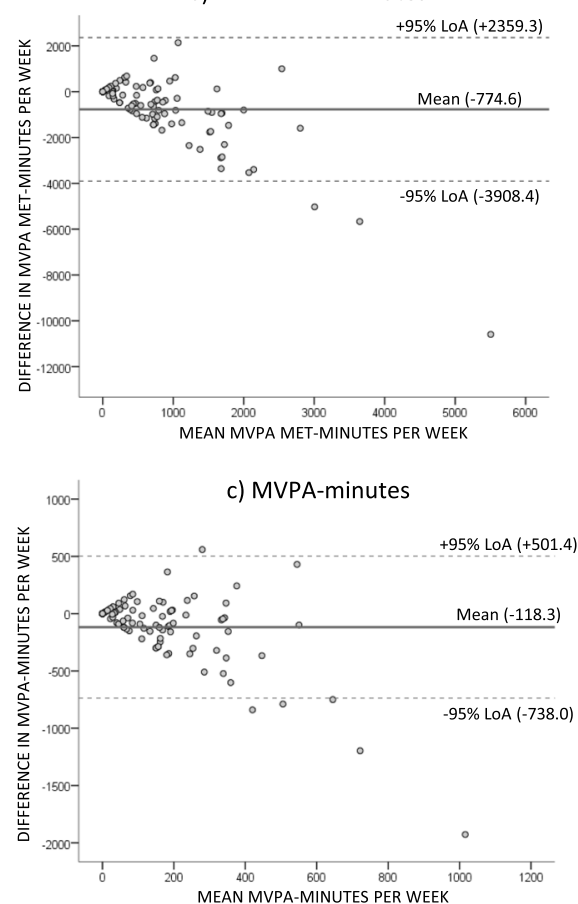

d) Vigorous PA-minutes

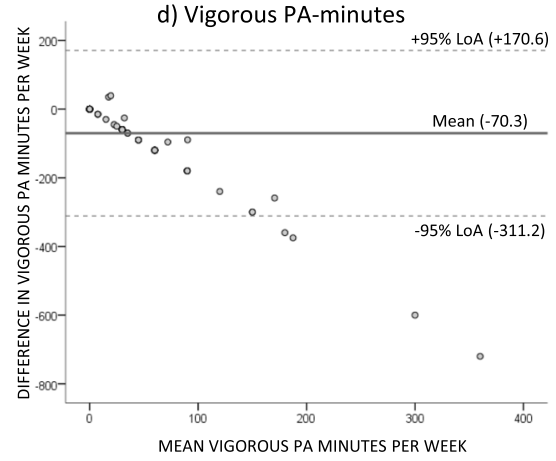

e) Moderate PA-minutes
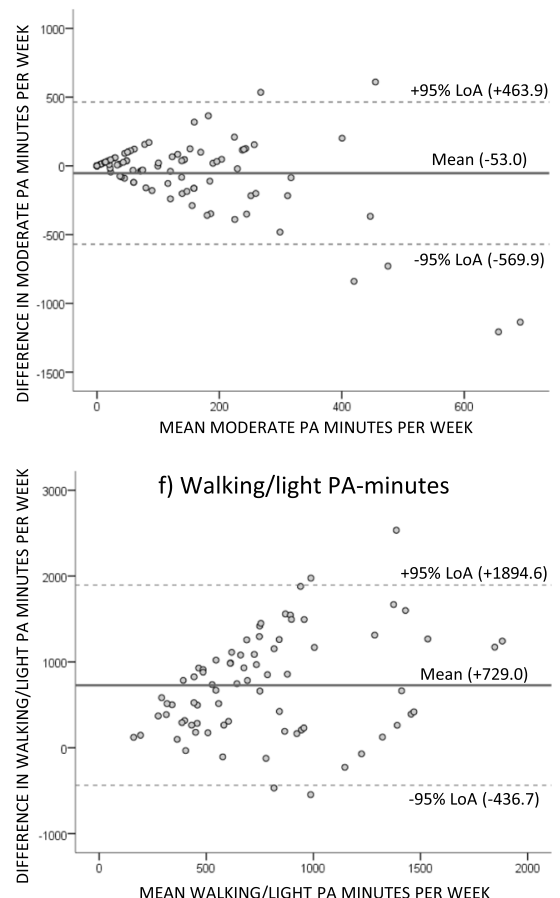

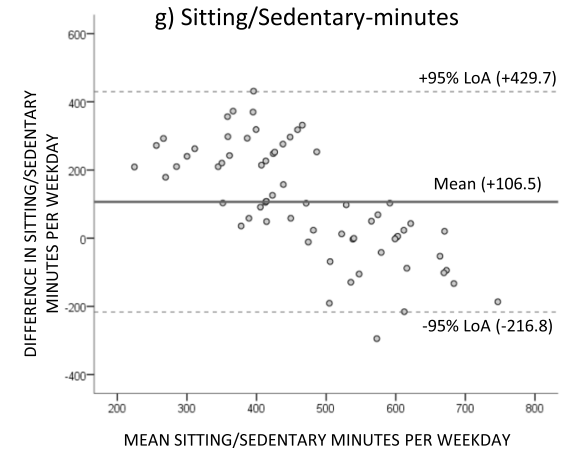

Fig. 2 Bland-Altman plots of the agreement between self-reported PA (IPAQ-SF) and accelerometer measured PA illustrated with mean difference and 95\% Limits of Agreement (LoA)

recommendations. Additionally, most of the included patients were women, and the validity of the results for men with OA is therefore unclear. However, the majority of patients with OA are women $[29,34,35]$, and the results may thereby be valid for the population seen in clinical practice. Further, we targeted a sample of 100 patients and 93 patient provided sufficient PA data. In rating of methodological quality of studies on 
measurement properties it is suggested that samples of 50 is good and 100 is excellent [24]. In our study we analyzed a sample close to 100 , and we found no differences in any demographic characteristics between those with and without sufficient PA data. Lastly, the accelerometer used as a criterion-method is not validated in patients with OA [36]. Considering that resting metabolic rate, gender, BMI, and work economy are shown to affect the accuracy of accelerometer measured PA [37, 38], combining accelerometry with heart rate monitoring may improve the accuracy in estimating energy expenditure of habitual PA $[39,40]$. Future studies should investigate the accelerometers accuracy in classifying PA intensity in patients with $\mathrm{OA}$, including different subgroups (i.e. OA phenotypes, gender, BMI, age). This is important knowledge in the search for optimal PA dosages in the treatment of OA. But, in lack of a true gold standard to measure habitual PA, accelerometry may be considered the best available single method.

In our study, we found that the patients reported a three-fold more time in high-intensity PA than what accelerometer recordings showed for the same days. An explanation for this could be that experienced intensity may be inflated due to OA symptoms like pain, fatigue or functional limitations. It is previously shown that responses on OA-specific questionnaires are strongly correlated with patients' pain-level, while performance based tests are less influenced by pain [41, 42]. Accordingly, self-report and objective measures of PA do not necessarily provide similar, but rather complementary information, and both methods are needed to better understand the performance and experience of activity in patients with OA. Even if the accelerometer gives more accurate data on habitual activity, the self-report method may capture the patient's experience of being active. This is valuable information for the clinician in helping patients to overcome barriers and motivate for activity as part of the disease management.

\section{Conclusion}

We found that correlations between the objective criterion-method and the self-reporting in IPAQ-SF were weak. However, self-reporting PA may capture the patients' experienced intensity of physical activity, which is important for clinicians in providing an optimal PA treatment program. Physical activity dosed according to guidelines is the most important treatment of $\mathrm{OA}$, and the finding that patients with OA tend to over-report activity of higher intensity and under-report low-intensity activity and sitting-time is therefore of clinical importance.

\section{Abbreviations}

BMI: Body Mass Index; Cl: Confidence Interval; CPM: Counts Per Minute; IPAQSF: International Physical Activity Questionnaire-Short Form; IQR: Interquartile
Range; MET: Metabolic Equivalent of Task; MVPA: Moderate to Vigorous intensity Physical Activity; NRS: Numeric Rating Scale; OA: Osteoarthritis; PA: Physical Activity; SD: Standard Deviation

\section{Acknowledgements}

The authors thank all patients for participating, and the osteoarthritis-course administrator (Liv Skancke and Liv Rognerud Eriksson) for facilitating recruitment of patients.

\section{Authors' contributions}

All authors (KLJ, HD, AC, KBH, ATT) made substantial contributions to the study conception and design, drafting and critical revision of the article, and final approval of the submitted version. KLJ and AC contributed to the data acquisition, and $\mathrm{KLJ}, \mathrm{HD}$ and $\mathrm{ATT}$ were responsible for data analyses and interpretation.

\section{Authors' information}

$\mathrm{KLJ}(\mathrm{MSC}), \mathrm{HD}(\mathrm{PhD}, \mathrm{PT}), \mathrm{AC}(\mathrm{PhD}, \mathrm{PT}), \mathrm{KBH}(\mathrm{PhD}, \mathrm{PT}), \mathrm{ATT}(\mathrm{PhD}, \mathrm{PT})$.

\section{Funding}

The study was financially supported by Diakonhjemmet Hospital AS (Oslo, Norway) as employer of the authors (KL, HD, AC, KBH and ATT).

Accelerometers were acquired by financial support granted by the Klaveness Foundation. The authors of the study initiated and completed the research independently of parties providing financial support.

\section{Availability of data and materials}

The datasets used and analysed during the current study are available from the corresponding author on reasonable request.

\section{Ethics approval and consent to participate}

The study was carried out in compliance with the Declaration of Helsinki and was evaluated by the Regional Committee for Medical and Health Research Ethics (2017/1559) and approved by a Data Protection Officer at Oslo University Hospital (17/16918). Eligible patients were given verbal- and written information about the study, and those providing written, informed consent were included.

\section{Consent for publication}

Not applicable.

\section{Competing interests}

The authors declare that they have no competing interests.

\section{Author details}

${ }^{1}$ National Advisory Unit on Rehabilitation in Rheumatology, The Division of Rheumatology and Research, Diakonhjemmet Hospital, Oslo, Norway.

${ }^{2}$ Faculty of Medicine, Institute of Health and Society, University of Oslo, Oslo, Norway. ${ }^{3}$ Division of Health Service, Norwegian Institute of Public Health, Oslo, Norway.

Received: 30 September 2020 Accepted: 10 February 2021 Published online: 27 February 2021

\section{References}

1. Rausch Osthoff AK, Niedermann K, Braun J, Adams J, Brodin N, Dagfinrud $H$, et al. 2018 EULAR recommendations for physical activity in people with inflammatory arthritis and osteoarthritis. Ann Rheum Dis 2018;77(9):1251-60.

2. Geenen $\mathrm{R}$, Overman $\mathrm{CL}$, Christensen $\mathrm{R}$, Asenlof $\mathrm{P}$, Capela $\mathrm{S}$, Huisinga $\mathrm{KL}$, et al. EULAR recommendations for the health professional's approach to pain management in inflammatory arthritis and osteoarthritis. Ann Rheum Dis. 2018;77(6):797-807.

3. Kendzerska T, Juni P, King LK, Croxford R, Stanaitis I, Hawker GA. The longitudinal relationship between hand, hip and knee osteoarthritis and cardiovascular events: a population-based cohort study. Osteoarthritis and cartilage / OARS, Osteoarthritis Research Society. 2017;25(11):1771-80.

4. Fernandes GS, Valdes AM. Cardiovascular disease and osteoarthritis: common pathways and patient outcomes. Eur J Clin Investig. 2015:45(4): 405-14. 
5. Swain S, Sarmanova A, Coupland C, Doherty M, Zhang W. Comorbidities in osteoarthritis: a systematic review and meta-analysis of observational studies. Arthritis Care Res. 2019.

6. Juhl C, Christensen R, Roos EM, Zhang W, Lund H. Impact of exercise type and dose on pain and disability in knee osteoarthritis: a systematic review and meta-regression analysis of randomized controlled trials. Arthritis Rheumatol. 2014;66(3):622-36.

7. Moseng T, Dagfinrud H, Smedslund G, Østerås N. The importance of dose in land-based supervised exercise for people with hip osteoarthritis. A systematic review and meta-analysis. Osteoarthritis and cartilage / OARS, Osteoarthritis Research Society. 2017;25(10):1563-76.

8. Moseng T, Dagfinrud H, Smedslund G, Østerås N. Corrigendum to 'The importance of dose in land-based supervised exercise for people with hip osteoarthritis. A systematic review and meta-analysis' [osteoarthritis cartilage 25 (2017) 1563-1576]. Osteoarthritis and cartilage / OARS, Osteoarthritis Research Society. 2018;26(5):707-9.

9. Trost SG, O'Neil M. Clinical use of objective measures of physical activity. $\mathrm{Br}$ J Sports Med. 2014;48(3):178-81.

10. Caspersen CJ, Powell KE, Christenson GM. Physical activity, exercise, and physical fitness: definitions and distinctions for health-related research. Public health reports (Washington, DC : 1974). 1985;100(2):126-31.

11. Westerterp KR. Assessment of physical activity: a critical appraisal. Eur J Appl Physiol. 2009;105(6):823-8.

12. Westerterp KR. Reliable assessment of physical activity in disease: an update on activity monitors. Curr Opin Clin Nutr Metab Care. 2014;17(5):401-6.

13. John D, Freedson P. ActiGraph and Actical physical activity monitors: a peek under the hood. Med Sci Sports Exerc. 2012;44(1 Suppl 1):S86-9.

14. Plasqui $G$, Bonomi AG, Westerterp KR. Daily physical activity assessment with accelerometers: new insights and validation studies. Obes Rev. 2013: 14(6):451-62.

15. Plasqui G, Westerterp KR. Physical activity assessment with accelerometers: an evaluation against doubly labeled water. Obesity (Silver Spring). 2007; 15(10):2371-9

16. Helmerhorst HJ, Brage S, Warren J, Besson H, Ekelund U. A systematic review of reliability and objective criterion-related validity of physical activity questionnaires. Int J Behav Nutr Phys Act. 2012:9:103.

17. Durante R, Ainsworth BE. The recall of physical activity: using a cognitive model of the question-answering process. Med Sci Sports Exerc. 1996; 28(10):1282-91.

18. Terwee $\mathrm{CB}$, Bouwmeester W, van Elsland SL, de Vet HC, Dekker J. Instruments to assess physical activity in patients with osteoarthritis of the hip or knee: a systematic review of measurement properties. Osteoarthritis and cartilage / OARS, Osteoarthritis Research Society. 2011;19(6):620-33.

19. Lee PH, Macfarlane DJ, Lam TH, Stewart SM. Validity of the International Physical Activity Questionnaire Short Form (IPAQ-SF): a systematic review. Int J Behav Nutr Phys Act. 2011:8:115.

20. Smith RD, Dziedzic KS, Quicke JG, Holden MA, McHugh GA, Healey EL. Identification and evaluation of self-report physical activity instruments in adults with osteoarthritis: a systematic review. Arthritis care \& research. 2019;71(2):237-51

21. Healey EL, Allen KD, Bennell K, Bowden JL, Quicke JG, Smith R. Self-Report Measures of Physical Activity. Arthritis care \& research. 2020;72(Suppl 10): 717-30

22. Craig $C L$, Marshall AL, Sjostrom M, Bauman AE, Booth ML, Ainsworth BE, et al. International physical activity questionnaire: 12 -country reliability and validity. Med Sci Sports Exerc. 2003;35(8):1381-95.

23. Vet HCWd, Terwee CB, Mokking LB, Knol DL. Measurement in medicine : a practical guide. Cambridge: Cambridge University Press; 2011.

24. Terwee CB, Mokkink LB, Knol DL, Ostelo RW, Bouter LM, de Vet HC. Rating the methodological quality in systematic reviews of studies on measurement properties: a scoring system for the COSMIN checklist. Qual Life Res. 2012;21(4):651-7.

25. Troiano RP, Berrigan D, Dodd KW, Masse LC, Tilert T, McDowell M. Physical activity in the United States measured by accelerometer. Med Sci Sports Exerc. 2008;40(1):181-8.

26. Dyrstad SM, Hansen BH, Holme IM, Anderssen SA. Comparison of selfreported versus accelerometer-measured physical activity. Med Sci Sports Exerc. 2014:46(1):99-106.

27. Schober P, Boer C, Schwarte LA. Correlation coefficients: appropriate use and interpretation. Anesth Analg. 2018;126(5):1763-8.
28. Blikman T, Stevens M, Bulstra SK, van den Akker-Scheek I, Reininga IH. Reliability and validity of the Dutch version of the international physical activity questionnaire in patients after total hip arthroplasty or total knee arthroplasty. The Journal of orthopaedic and sports physical therapy. 2013; 43(9):650-9

29. Liu SH, Eaton CB, Driban JB, McAlindon TE, Lapane KL. Comparison of selfreport and objective measures of physical activity in US adults with osteoarthritis. Rheumatol Int. 2016;36(10):1355-64.

30. Bland JM, Altman DG. Measuring agreement in method comparison studies. Stat Methods Med Res. 1999:8(2):135-60.

31. Svege I, Kolle E, Risberg MA. Reliability and validity of the physical activity scale for the elderly (PASE) in patients with hip osteoarthritis. BMC Musculoskelet Disord. 2012;13:26

32. Herman Hansen B, Bortnes I, Hildebrand M, Holme I, Kolle E, Anderssen SA. Validity of the ActiGraph GT1M during walking and cycling. J Sports Sci. 2014;32(6):510-6.

33. Kodama S, Saito K, Tanaka S, Maki M, Yachi Y, Asumi M, et al. Cardiorespiratory fitness as a quantitative predictor of all-cause mortality and cardiovascular events in healthy men and women: a meta-analysis. Jama. 2009;301(19):2024-35.

34. Pelle T, Claassen A, Meessen J, Peter WF, Vliet Vlieland TPM, Bevers K, et al. Comparison of physical activity among different subsets of patients with knee or hip osteoarthritis and the general population. Rheumatol Int. 2020; 40(3):383-92.

35. Herbolsheimer F, Schaap LA, Edwards MH, Maggi S, Otero Á, Timmermans EJ, et al. Physical activity patterns among older adults with and without knee osteoarthritis in six European countries. Arthritis care \& research. 2016; 68(2):228-36

36. Sliepen M, Brandes M, Rosenbaum D. Current physical activity monitors in hip and knee osteoarthritis: a review. Arthritis care \& research. 2017;69(10): 1460-6.

37. Aadland E, Steene-Johannessen J. The use of individual cut points from treadmill walking to assess free-living moderate to vigorous physical activity in obese subjects by accelerometry: is it useful? BMC Med Res Methodol. 2012;12:172.

38. Barnett A, van den Hoek D, Barnett D, Cerin E. Measuring moderateintensity walking in older adults using the ActiGraph accelerometer. BMC Geriatr. 2016;16(1):211

39. Strath SJ, Brage S, Ekelund U. Integration of physiological and accelerometer data to improve physical activity assessment. Med Sci Sports Exerc. 2005; 37(11 Suppl):S563-71.

40. Brage S, Westgate K, Franks PW, Stegle O, Wright A, Ekelund U, et al. Estimation of free-living energy expenditure by heart rate and movement sensing: a doubly-Labelled water study. PLoS One. 2015;10(9):e0137206.

41. Terwee CB, van der Slikke RM, van Lummel RC, Benink RJ, Meijers WG, de Vet HC. Self-reported physical functioning was more influenced by pain than performance-based physical functioning in knee-osteoarthritis patients. J Clin Epidemiol. 2006:59(7):724-31.

42. Stratford PW, Kennedy DM. Performance measures were necessary to obtain a complete picture of osteoarthritic patients. J Clin Epidemiol. 2006;59(2): 160-7.

\section{Publisher's Note}

Springer Nature remains neutral with regard to jurisdictional claims in published maps and institutional affiliations.

\section{Ready to submit your research? Choose BMC and benefit from:}

- fast, convenient online submission

- thorough peer review by experienced researchers in your field

- rapid publication on acceptance

- support for research data, including large and complex data types

- gold Open Access which fosters wider collaboration and increased citations

- maximum visibility for your research: over $100 \mathrm{M}$ website views per year

At BMC, research is always in progress.

Learn more biomedcentral.com/submission 